\title{
Baroclinic waves in an air-filled thermally driven rotating annulus
}

\author{
A. A. Castrejón-Pita and P. L. Read \\ Atmospheric, Oceanic and Planetary Physics, Clarendon Laboratory, University of Oxford, \\ Parks Road, Oxford, OX1 3PU, United Kingdom
}

(Received 18 July 2006; published 5 February 2007)

\begin{abstract}
In this study an experimental investigation of baroclinic waves in air in a differentially heated rotating annulus is presented. Air has a Prandtl number of 0.707 , which falls within a previously unexplored region of parameter space for baroclinic instability. The flow regimes encountered include steady waves, periodic amplitude vacillations, modulated amplitude vacillations, and either monochromatic or mixed wave number weak waves, the latter being characterized by having amplitudes less than $5 \%$ of the applied temperature contrast. The distribution of these flow regimes in parameter space are presented in a regime diagram. It was found that the progression of transitions between different regimes is, as predicted by recent numerical modeling results, in the opposite sense to that usually found in experiments with high Prandtl number liquids. No hysteresis in the flow type, with respect to variations in the rotation rate, was found in this investigation.
\end{abstract}

DOI: 10.1103/PhysRevE.75.026301

PACS number(s): 47.32.Ef, 47.20.Ft, 47.20.Bp

\section{INTRODUCTION}

Baroclinic waves have an important role in the middle latitude circulation of the atmosphere, contributing strongly to poleward heat transport in the form of baroclinic waves or eddies. These are some of the most energetic components of the atmospheric circulation and are responsible for major meteorological disturbances such as cyclones and anticyclones or other weather phenomena on the Earth or on other planets [1-3]. Numerous studies, both theoretical and experimental, have been carried out to try to understand this instability and its consequences in the form of fully developed baroclinic waves [4-7]. The archetypical experiment used to study this phenomenon is the thermally driven, rotating, cylindrical annulus. This experiment has been used for many years to demonstrate, on a small scale and under controlled, repeatable conditions, the development of baroclinic waves $[5,6,8]$. It has been found that the flow in the annulus experiment can exhibit a rich variety of regimes, depending on the fluid and on the parameters used to perform the experiment, such as the rotation rate, $\Omega$, temperature contrast, $\Delta T$, aspect ratios, viscosity, thermal diffusivity and density of the fluid. The different regimes that can be obtained in such an experiment include axisymmetric flows, fully developed steady baroclinic waves, waves with periodic amplitude modulations or vacillations (AV), quasiperiodic or chaotically modulated amplitude vacillations (MAV), other forms of so-called structural vacillations (SV), and irregular flow (a form of geostrophic turbulence). Despite the variety of experimental studies carried out using different fluids with different properties and with a relatively high, or low Prandtl number [9] (i.e., $\geqslant 5$ or $\leqslant 0.025$ ), the intermediate range, $O(1)$, has not so far been experimentally studied for this problem.

A recent investigation [10] has provided a detailed analysis of the onset of baroclinic waves in an air-filled rotating baroclinic annulus using a three-dimensional direct numerical simulation. The results are analyzed in the context of a bifurcation analysis using nonlinear time series analysis techniques, including spectral analysis, phase portrait reconstruction, and Lyapunov exponent estimations. The transition curve, or regime diagram, shows the typical anvil-like shape in the parameter plane, in agreement with previously published material using a diverse variety of liquids with different Prandtl numbers. In particular, the investigation was also focused on the investigation of the bifurcation sequence from axisymmetric flows to more complex flows. They found that, as the rotation rate was increased, this sequence progressed from axisymmetric flow to fully developed baroclinic waves of constant amplitude. This was followed by two more bifurcations, first to a periodic amplitude vacillation, then to a quasiperiodic MAV, and finally to a chaotic MAV regime. This is, in certain respects, in disagreement with previous findings from investigations of the baroclinic annulus with liquids, where the onset of amplitude vacillation was found to occur only on decreasing the rotation rate. This observation suggests that the value of the Prandtl number, which implies changes in the thickness and structure of the thermal and velocity boundary layers, are influential in determining the direction in parameter space for transitions between axisymmetric and vacillating flows on variation of the rotation rate $\Omega$ or other parameters. However, there are no published experimental studies of an air-filled annulus to corroborate these findings. Motivated partly by these numerical studies, the present paper presents an experimental study of baroclinic waves in a rotating annulus using air as the working fluid, which has a Prandtl number of 0.707 , well inside the previously unexplored region of parameter space. Although the dimensions of the annulus used in this work are not quite the same as used in the numerical simulations presented by [10], it is of interest (not least as an indication of their robustness) to examine whether the main aspects of the dynamical bifurcations are at least qualitatively consistent with these numerical results. The annular container used in the numerical simulation presented in [10] had an inner cylinder with a radius $a=34.8 \mathrm{~mm}$, the outer cylinder had a radius $b=60.2 \mathrm{~mm}$, and a depth of $d=100 \mathrm{~mm}$, based on the experiments presented of [11].

The main objective of this paper, therefore, is to present the results of investigations performed using an air-filled rotating annulus experiment. Section II provides a description of the two different experimental rigs used to perform the 


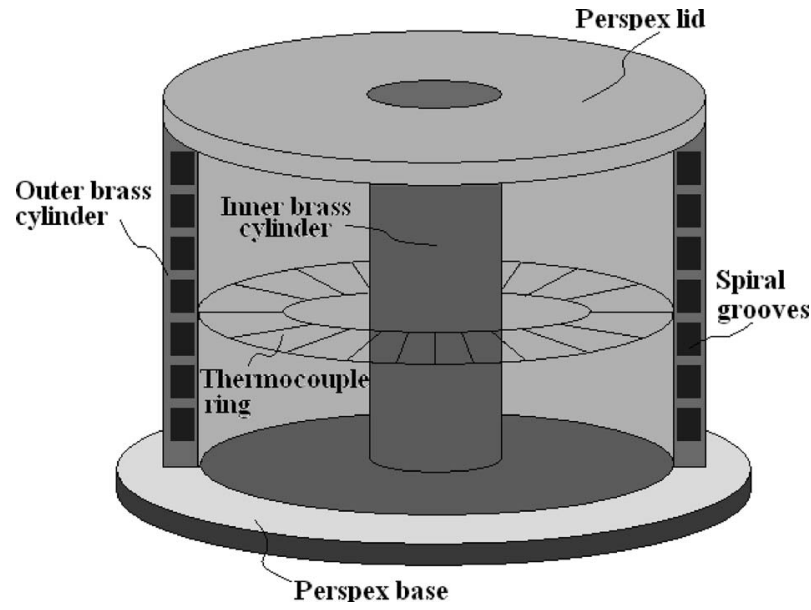

FIG. 1. Experimental setup 1-temperature measurement.

investigation. One of them was used to perform temperature measurements while another enabled flow visualization. The next section, Sec. III, briefly describes the spectral and other diagnostic methods used in the analysis of the results. Section IV presents both general and particular results for each of the main flow types found in the experiment. Finally, in Sec. $\mathrm{V}$ a discussion of the results is presented.

\section{EXPERIMENTAL SETUP}

\section{A. Temperature measurement facility}

The experimental setup consisted of an upright cylindrical annular container mounted on a turntable base so that it could be rotated about its vertical axis of symmetry. The annulus comprised two coaxial brass cylinders, each with a wall thickness of $2.5 \mathrm{~mm}$, while the lid and the base were made of perspex. The inner cylinder had a radius $a=25 \mathrm{~mm}$, the outer cylinder had a radius $b=80 \mathrm{~mm}$, and the depth of the annulus was $d=140 \mathrm{~mm}$. Inside the annular channel, at mid-depth and midradius, there was a copperconstantan thermocouple ring providing 32 measurement points, equally spaced in the azimuthal direction. A schematic view of this device is presented in Fig. 1.

The annulus was placed inside a temperature controlled enclosure in order to maintain a stable thermal environment. The temperature of the laboratory was also maintained constant to within $\pm 1.5^{\circ} \mathrm{C}$. The inner cylinder was typically cooled and the outer was warmed. The temperature of the inner and outer cylinders was regulated by water flowing in spiral grooves inside the inner cylinder and outside the outer cylinder. The circulating water in both circuits was first cooled by two external refrigerators for the inner cylinder and one refrigerator on the turntable for the outer cylinder. The final correction to the temperature was achieved by warming the circulating water using heaters on the turntable controlled by a computerized temperature controller (a Eurotherm model 2704). This allowed the coolant water temperature to be maintained to within a precision of $\pm 0.02{ }^{\circ} \mathrm{C}$. The temperature of the walls, the environment enclosure, and that of the laboratory were also acquired from additional thermo- couple sensors at regular intervals and saved for further analysis.

The temperature measurements provided by the thermocouples were digitized by a Schlumberger 3531F data acquisition system (DAQ) in the rotating frame. These data were then transferred in real time to a PC in the stationary laboratory frame. The rotation of the turntable was produced by a servocontrolled d.c. motor controlled by the DAQ via a feedback loop. The system is capable of controlling the rotation rate with a precision of $\pm 0.0004 \mathrm{rad} / \mathrm{s}$. For a more detailed description of the experimental facility see $[4,8]$. The maximum data acquisition rate had a period of six seconds.

From multiple short runs, it was found that one hour was long enough for the development and equilibration of baroclinic waves at low rotation rates (less than $3 \mathrm{rad} / \mathrm{s}$ ). For high rotation rates, where large amplitude baroclinic waves are present, the experiments were performed for two or three hours. The experiments were performed in two ways: increasing the rotation rate gently (from $1 \mathrm{rad} / \mathrm{s}$ up to $4.5 \mathrm{rad} / \mathrm{s}$ in steps), and descending (starting at $0 \mathrm{rad} / \mathrm{s}$, abruptly jumping to $4.5 \mathrm{rad} / \mathrm{s}$, and then descending in steps down to $1.5 \mathrm{rad} / \mathrm{s}$ ) for each applied $\Delta T$. This was done in order to find out if the system exhibited hysteresis to rotation rate changes.

\section{B. Flow visualization facility}

A second annulus was used to perform flow visualization. It used almost the same configuration as described above with the difference that the outer cylinder had a set of transparent perspex rings inserted at five heights, namely, $h_{1}=16 \mathrm{~mm}, h_{2}=43 \mathrm{~mm}, h_{3}=70 \mathrm{~mm}, h_{4}=97 \mathrm{~mm}$, and $h_{5}=124 \mathrm{~mm}$ from the bottom. These inserts were designed to allow collimated horizontal light sheets (parallel to the horizontal endwalls) of thickness $\sim 2.5 \mathrm{~mm}$ to illuminate the annular space at these five different levels. The light sheet illuminated suspended microparticles in the air. The seeding particles used were micro-oil droplets with an approximate radius of $1 \mu \mathrm{m}$ produced by high-temperature evaporation and condensation of an oil-water-based solution, using a commercial smoke and/or fog machine. The smoke machine generated a dense white fog which was transferred inside the annular container through a hose at the beginning of the experiment. The oil droplet fog's durability in air before it sediments was about 20-30 min, imposing a time limit on the duration of the visualization experiments. The usual time for the development of the waves was between 5 and $10 \mathrm{~min}$. Thus, after the injection of the smoke into the annulus, the experiments needed to be carried out as soon as possible, due to the time restriction. The high intensity light sheets were created by collimating light provided by three (per level) $50 \mathrm{~W}$ quartz-halogen incandescent bulbs, located at equally spaced angles around the outside of the tank. The thickness of the light sheet was approximately $3 \mathrm{~mm}$. The light scattered by the particles was captured by a video camera mounted on the turntable over the annulus. A COHU high performance monochrome charge-coupled device (CCD) camera and a Sony digital camcorder (PC105) were used to capture images and videos. A schematic diagram of this facility is shown in Fig. 2. 

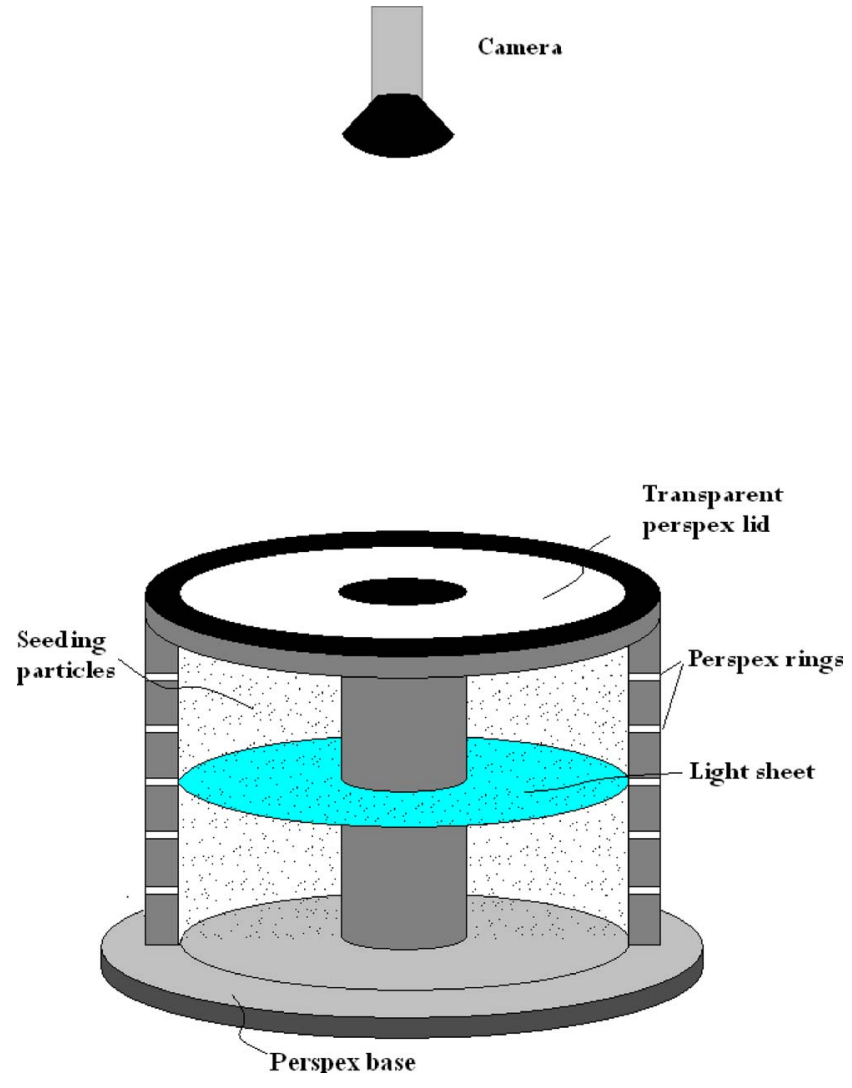

FIG. 2. (Color online) Experimental setup for flow visualization.

The temperature control systems were very similar to those described for the previous experimental setup, including water pumps, coolers, heaters and a Eurotherm temperature controller. The annulus, visualization system, the entire heating and video capture systems were mounted on the turntable. The experiment was controlled externally by a computer (in the laboratory frame) which allowed the variation of the rotation rate, $\Omega$, the temperature difference, $\Delta T$, between the inner and the outer cylinder, and the visualization and/or light-sheet system. The video images were monitored in real time and recorded on digital video tape.

\section{DATA ANALYSIS}

The wave numbers, $m$, drift frequencies, $\omega_{d}$, vacillation frequencies, $\omega_{v}$, and modulated vacillation frequencies, $\omega_{m}$, were calculated by applying fast Fourier techniques (spatial and temporal FFTs) to the thermocouple data. A spatial Fourier transform was applied to the data provided by the thermocouple ring at each sample time in order to determine the dominant azimuthal wave number and its possible temporal amplitude variations, i.e., where the amplitude vacillation waves (AV) or modulated amplitude vacillation waves (MAV) were developing. The frequency of this oscillation was found by applying a temporal Fourier transform to the amplitude oscillation data. The drift frequency $\omega_{d}$ was also easily identified as the dominant frequency in a frequency spectrum calculated by applying a temporal Fourier trans-

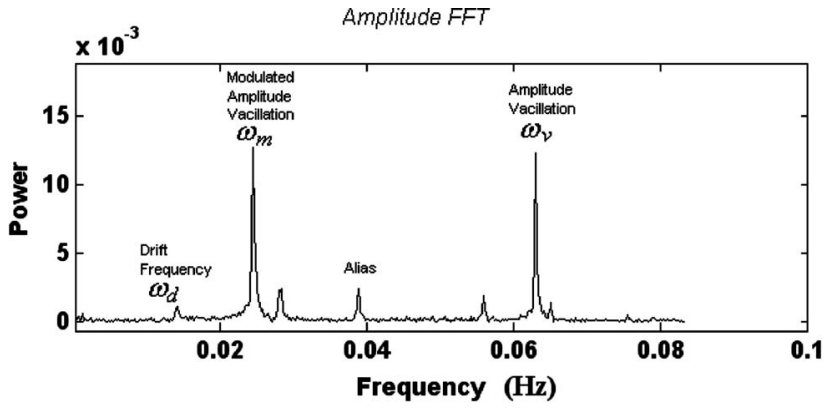

Phase FFT

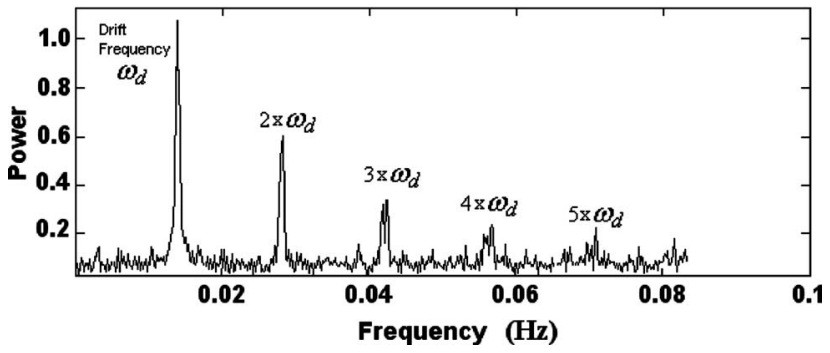

FIG. 3. Frequency spectrum for an MAV wave signal showing the drift, vacillation, modulated vacillation, and rotationally induced alias frequencies. $\Omega=2.327$ giving an aliased frequency, $\nu_{\text {alias }} \approx 0.037 \mathrm{~Hz}$.

form to the signal of a single thermocouple from the thermocouple ring. Similar analysis was applied to the data provided by thermocouples located at the annulus walls, inside the thermal enclosure, in the circulating water, and in the laboratory.

The analysis to identify the different frequencies was carried out very carefully. Due to the relatively sparse data sampling interval of $6 \mathrm{~s}$, it was considered likely that certain frequencies could be aliased, leading to spectral peaks that could be easily confused with other real and physically important frequencies. These alias frequencies are the result of under sampling a high-frequency signal (outside the Nyquist frequency range). The analysis of these alias frequencies was prompted by the appearance of certain peaks in the frequency spectrum of the temperature measurements of the thermocouple ring and in those acquired from thermocouples located outside the annulus itself, i.e., in the circulating water, the wall temperatures, and in the external environmental temperature. It was also found that the same unknown frequency appeared in all the experiments with the same rotation rate, no matter the temperature contrast used to perform the experiment. This suggested that these frequencies were not due to oscillations or waves in the convecting fluid, but were produced by other external effects. Consequently, an identification of the source of this noise had to be found. In this case, these aliased frequencies were likely to have been produced by electromagnetic interference produced from the magnetic fields produced by the dc motor that maintains the rotation of the turntable. These electromagnetic signals are thought to have affected the data acquisition, producing interference at frequency $\Omega$ itself in the saved data.

The corresponding aliased frequencies in the discretely sampled spectrum can be found from the relation 


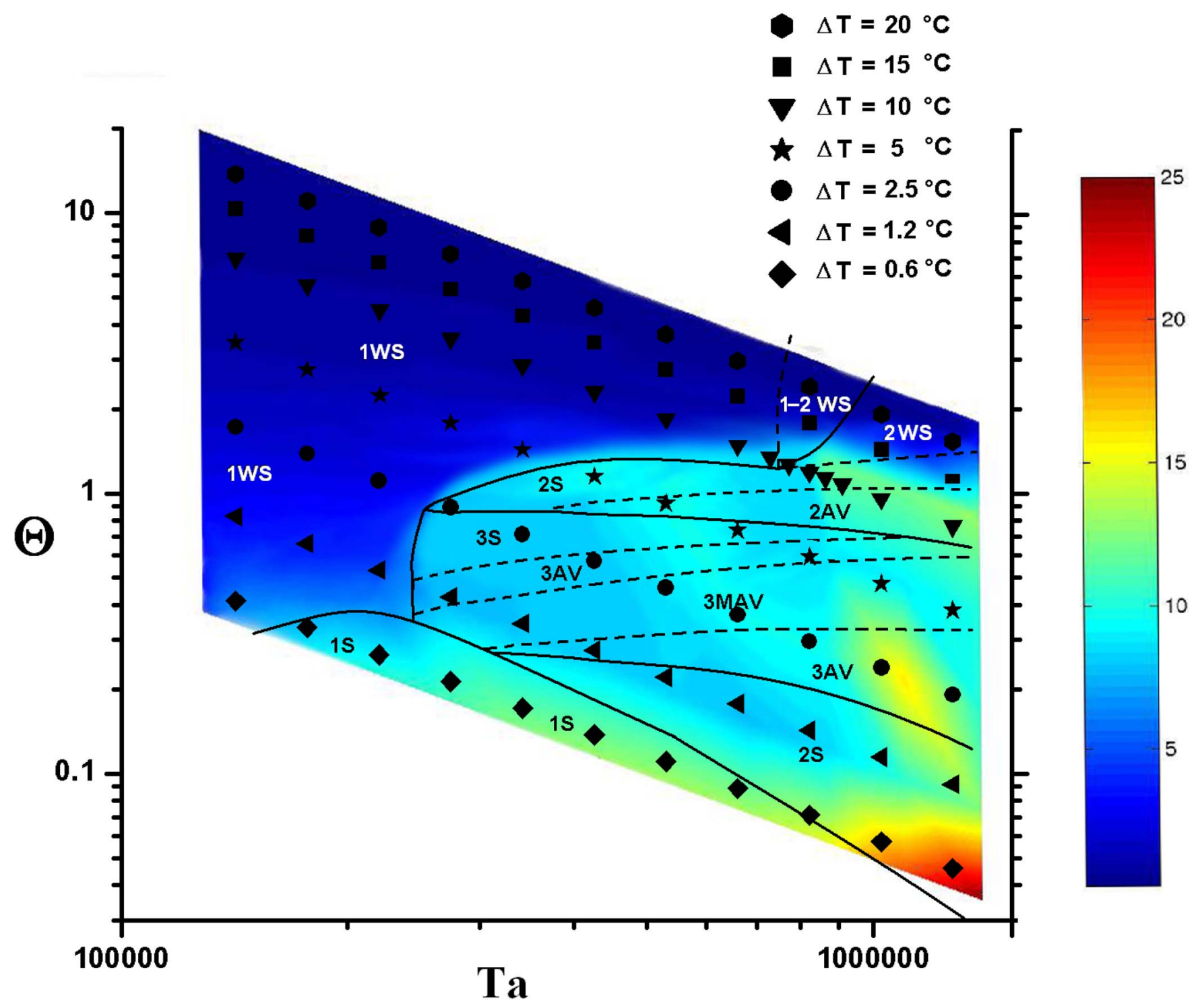

FIG. 4. (Color online) Regime diagram. The dominant azimuthal wave number, $m$, is indicated by a number, the flow type is indicated with letters. Numbers designated $S$ denote a steady wave state, $W$ indicates a weak wave, AV denotes amplitude vacillation, and MAV modulated amplitude vacillations. Transitions between different wave numbers are shown with solid lines, whereas transitions between regimes of the same wave number are denoted by dotted lines. The corresponding normalized wave amplitude, $A_{n}$, is represented by shaded contours $\left(A_{n}=A_{w} / \Delta T\right.$, where $A_{w}$ is the wave amplitude in $\left.{ }^{\circ} \mathrm{C}\right)$.

$$
\nu_{\text {alias }}=\Omega / 2 \pi-n \nu_{\text {acq }} \text {, }
$$

where $\Omega$ is the turntable rotation rate (in $\operatorname{rad~s}^{-1}$ ), $\nu_{a c q}$ is the maximum nonaliased frequency (in $\mathrm{Hz}$ ) allowed to be identified with the given sampling rate (i.e., the Nyquist frequency), and $n$ is an integer that may need to be found empirically for each case such that the aliased frequency is shifted below $\nu_{a c q}$. In all the experiments the sampling period was $6 \mathrm{~s}$, so that $\nu_{a c q}=0.08333 \mathrm{~Hz}$.

The aliased frequencies corresponding to $\Omega$ for each experimental run were calculated, and were found to correspond perfectly (to within the experimental error) with identifiable peaks found in the frequency spectra of the temperature measurements mentioned above. An example of a typical frequency spectrum can be seen in Fig. 3 .

\section{RESULTS}

One of the main aims of the present work is to obtain a detailed experimental regime diagram for the air-filled annulus and to compare the results for particular flow regimes with those found numerically by Randriamampianina et al. [10]. There are two dimensionless parameters (in addition to the Prandtl number, Pr) commonly used to classify the dynamics of baroclinic instabilities in a rotating thermally driven rotating annulus. These are the Taylor number, Ta, defined as

$$
\mathrm{Ta}=\frac{4 \Omega^{2}(b-a)^{5}}{\nu^{2} d},
$$

and the thermal Rossby number, $\Theta$ (sometimes called the stability parameter or hide number), 

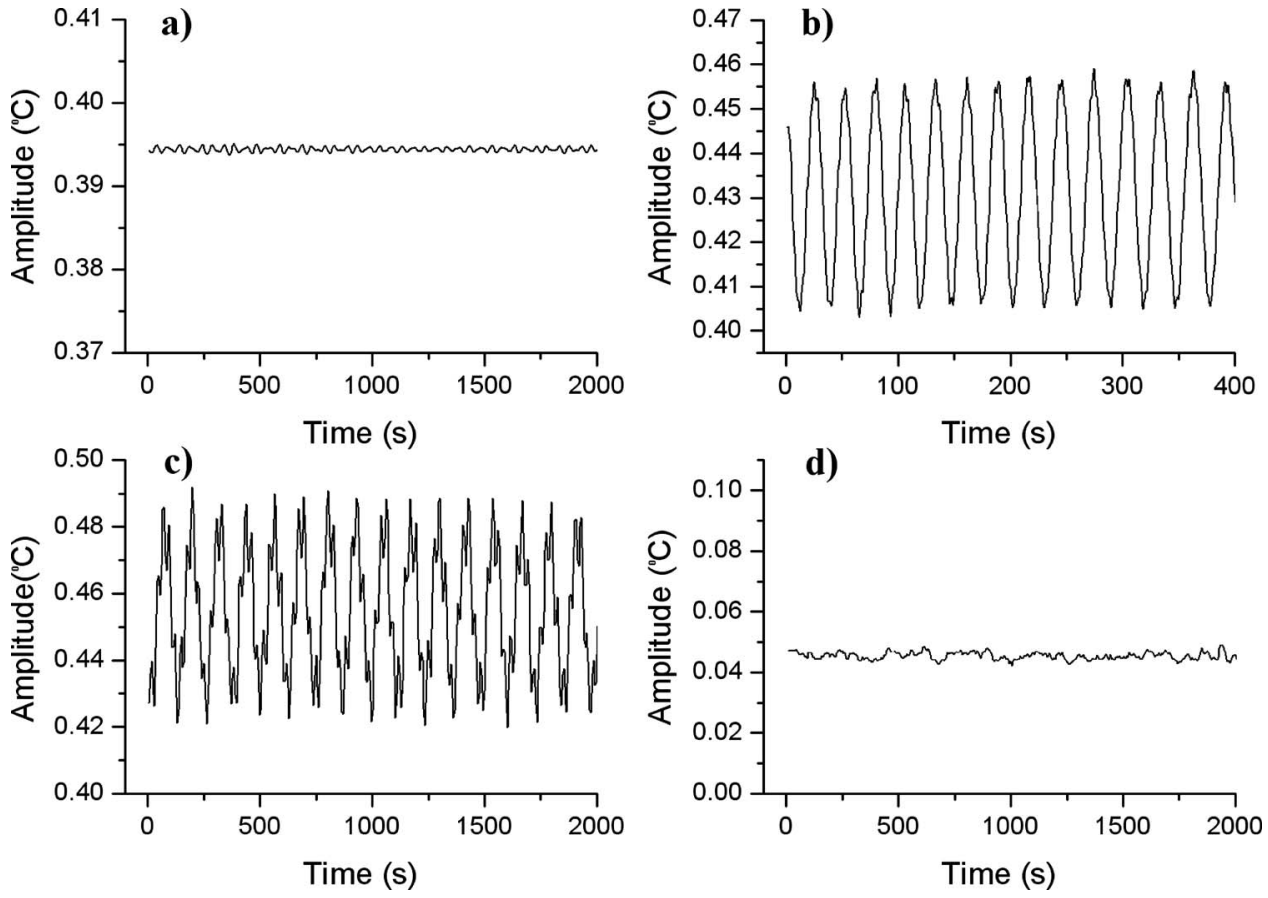

FIG. 5. Time variation of the temperature amplitude of the dominant azimuthal wave number for the four types of flow: (a) Steady flow, $2 S$ wave, $\mathrm{Ta}=4.26 \times 10^{5}$ and $\Theta=1.15$; (b) amplitude vacillation, 3 AV wave, $\mathrm{Ta}=8.23 \times 10^{5}$ and $\Theta=0.593$; (c) modulated amplitude vacillation, 3 MAV wave, $\mathrm{Ta}=10.2 \times 10^{5}$ and $\Theta=0.48$; and (d) weak wave, $1 \mathrm{WS}, \mathrm{Ta}=2.202 \times 10^{5}$ and $\Theta=2.218$.

$$
\Theta=\frac{g(\Delta \rho / \rho) d}{\Omega^{2}(b-a)^{2}},
$$

where $a$ and $b$ are the inner and outer radii of the annulus, respectively, $d$ is the depth of the annulus, $\nu$ is the kinematic viscosity of the working fluid $\left(\simeq 1.51 \times 10^{-5} \mathrm{~m}^{2} \mathrm{~s}^{-1}\right.$ for air at $20{ }^{\circ} \mathrm{C}$ ), and $\rho$ is the mean density of the fluid between the cylinders. Assuming the perfect gas law for air, the following expression for the fractional density contrast can be obtained:

$$
\frac{\Delta \rho}{\rho}=2 \frac{\left(T_{\text {out }}-T_{\text {in }}\right)}{\left(T_{\text {out }}+T_{\text {in }}\right)},
$$

where $T_{\text {in }}$ and $T_{\text {out }}$ are the temperatures (in ${ }^{\circ} \mathrm{C}$ ) of the inner and outer cylinders respectively. Having defined these parameters, a regime diagram can be constructed by systematically exploring a wide range of parameter space in small steps, usually by keeping $\Delta T$ fixed and varying $\Omega$. The resulting regime diagram shows the different regions of parameter space in which various different flow types are observed. The complete regime diagram found in the present work, including runs in which $\Omega$ was either successively increased or decreased, is shown in Fig. 4. The range of behavior includes large amplitude steady waves (denoted $S$ ), amplitude vacillating $(\mathrm{AV})$ waves, and modulated amplitude vacillation (MAV).

As can be seen from the regime diagram, a rich variety of different flow types was found in this experiment. The regime diagram shown in Fig. 4 also includes color contours showing the wave amplitude normalized with respect to the applied temperature contrast $\left(A_{n}=A_{w} / \Delta T\right.$, where $A_{w}$ is the actual dimensional wave amplitude). An abrupt change in the normalized amplitude can be seen between the regions of large amplitude waves and adjacent regions characterized by what we have called "weak waves."
It is noteworthy that purely axisymmetric flows were only observed at values of $\Omega$ less than $1 \mathrm{rad} \mathrm{s}^{-1}$. For $\Omega=1 \mathrm{rad} \mathrm{s}^{-1}$ some signs of weak wave behavior started to appear. Elsewhere within the range of parameter space explored some sort of azimuthally traveling wave signal was present in all other cases. As is the case with high Prandtl number liquids [9], the main baroclinically active regimes were characterized by the presence of waves with a normalized amplitude of around $10 \%$. In some cases, however, the wave amplitude reached values near $20 \%$ of the applied temperature gradient, which is relatively unusual in experiments with high Pr liquids. A weak wave regime has also been observed with high Pr fluids [12], but only at relatively large values of Ta. The "weak waves" regime here seems to be

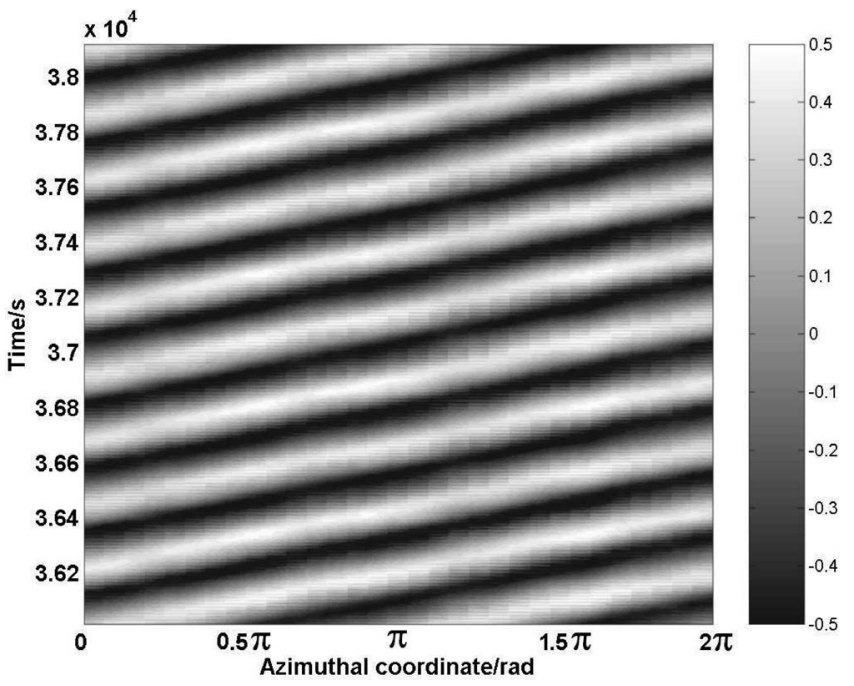

FIG. 6. Experimental azimuth-time temperature contour plot of a $2 S$ wave $\left(\mathrm{Ta}=4.26 \times 10^{5}\right.$ and $\left.\Theta=1.15\right)$ at midheight and midradius. 


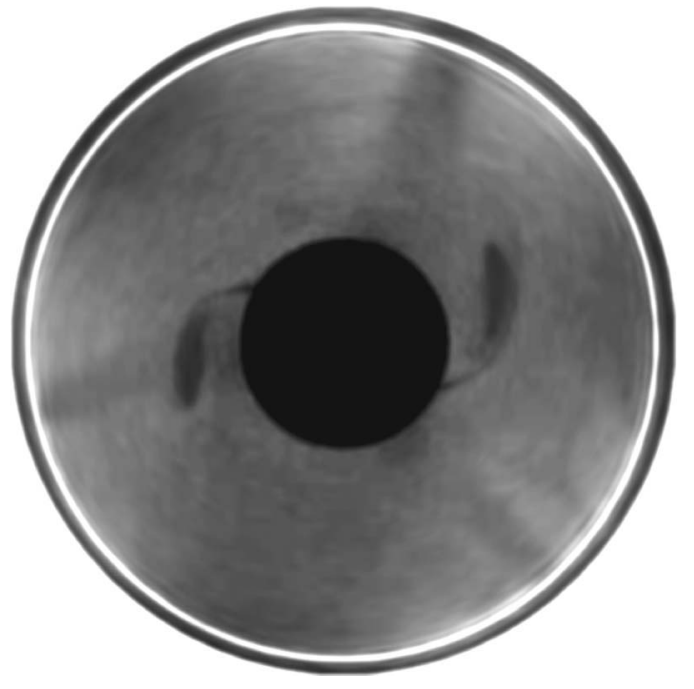

FIG. 7. Experimental visualization of a $2 S$ wave $\left(\mathrm{Ta}=4.26 \times 10^{5}\right.$ and $\left.\Theta=1.15\right)$ at midheight.

more ubiquitous and has properties which differ markedly from those found, e.g., by Hide and Mason [7], as will be discussed below. Another feature that is in contrast with previous investigations using high Prandtl number fluids is that in this work no hysteresis between regime transition was found when the rotation rate was varied. Some hysteresis was also predicted by the numerical studies of the air-filled baroclinic annulus [10].

This observation was considered robust because all the points in the regime diagram were studied by performing different runs where the rotation rate was either increased or decreased. The flow type found in both experiments at the same point in parameter space was the same. The wave number, $m$, was the same for both experiments and the difference in frequencies between both experiments was less than $3 \%$ for $\omega_{d}$, less than $5 \%$ for $\omega_{v}$, and less than $7 \%$ for $\omega_{m}$.

Figure 5 shows the time variation of the temperature amplitude of the dominant wave number, for the four main flow types found in this investigation for runs with $\Delta T=5^{\circ} \mathrm{C}$. Figure 5(a) clearly shows the absence of time dependence in the amplitude of steady waves, while Fig. 5(b) shows a periodic amplitude oscillation in the case of an AV flow. Figure 5 (c) is typical of a more complex MAV flow, and a steady weak wave is illustrated in Fig. 5(d).

Through the visualization experiments, it was possible to explore and observe the qualitative development of the baroclinic waves inside the annulus. Also it was used to verify the regime diagram obtained with the temperature measurement system. The weak wave flow was also found, confirming the results found using the temperature measurement facility. Again, a lack of hysteresis was detected and the progression of transitions between different wave number states was corroborated. Unfortunately, due to the small size of the seeding particles it was impossible to perform quantitative particle velocimetry.

\section{A. Steady wave regime}

The main steady wave regime is characterized by the presence of regular waves with a constant amplitude
TABLE I. Summary of general results for the experiment with $\Delta T=5{ }^{\circ} \mathrm{C}$ where $\Omega$ is the rotation rate, Ta is the Taylor number, $\Theta$ is the thermal Rossby number, $m$ is the wave number, $\omega_{d}$ is the drift frequency, $\omega_{v}$ is the vacillation frequency, $\omega_{m \nu}$ is the modulated amplitude vacillation, and $\eta$ is the vacillation index.

\begin{tabular}{lccccccc}
\hline \hline & $\mathrm{Ta}$ & & & & $\omega_{v}$ & $\omega_{m \nu}$ & \\
$\Omega$ & $\left(\times 10^{5}\right)$ & $\Theta$ & $m$ & $\omega_{d}(\mathrm{~Hz})$ & $(\mathrm{Hz})$ & $(\mathrm{Hz})$ & $\eta$ \\
\hline 1.5 & 1.42 & 3.44 & $1 W S$ & 0.0283 & - & - & - \\
1.674 & 1.76 & 2.76 & $1 W S$ & 0.0332 & - & - & - \\
1.868 & 2.20 & 2.22 & $1 W S$ & 0.0361 & - & - & - \\
2.085 & 2.74 & 1.78 & $1 W S$ & 0.0383 & - & - & - \\
2.327 & 3.42 & 1.43 & $1 W S$ & 0.0421 & - & - & - \\
2.598 & 4.26 & 1.15 & $2 \mathrm{~S}$ & 0.0043 & - & - & - \\
2.890 & 5.30 & 0.92 & $2 \mathrm{AV}$ & 0.0083 & 0.0304 & - & 0.102 \\
3.236 & 6.61 & 0.74 & $3 S$ & 0.0145 & - & - & - \\
3.610 & 8.23 & 0.59 & $3 \mathrm{AV}$ & 0.0198 & 0.0373 & - & 0.091 \\
4.000 & 10.2 & 0.48 & $3 \mathrm{MAV}$ & 0.0221 & 0.0382 & 0.008 & 0.087 \\
4.500 & 12.8 & 0.38 & $3 \mathrm{MAV}$ & 0.0227 & 0.0461 & 0.028 & 0.029 \\
\hline \hline
\end{tabular}

$\left(A_{n} \sim 0.1\right)$ and a small, constant drift velocity around the annulus. An example of this kind of wave flow is illustrated in Figs. 6 and 7. Figure 6 shows an azimuth-time temperature contour plot of a $2 S$ wave at midheight and midradius. Figure 7 shows a snapshot obtained from the visualization of the fluid, also at midheight and midradius. A time series of the amplitude of this wave is shown in Fig. 5(a), clearly demonstrating that the wave amplitude remains constant in time to within $\pm 0.002{ }^{\circ} \mathrm{C}$.

The maximum measured temperature amplitude in this example of a steady wave was $0.48 \pm 0.02{ }^{\circ} \mathrm{C}$, almost $10 \%$ of the applied temperature contrast. In some cases, for other parameters especially towards the lower right of the regime diagram at high Ta and low values of $\Theta$, the amplitude was found to approach $20 \%$ of the applied temperature gradient.

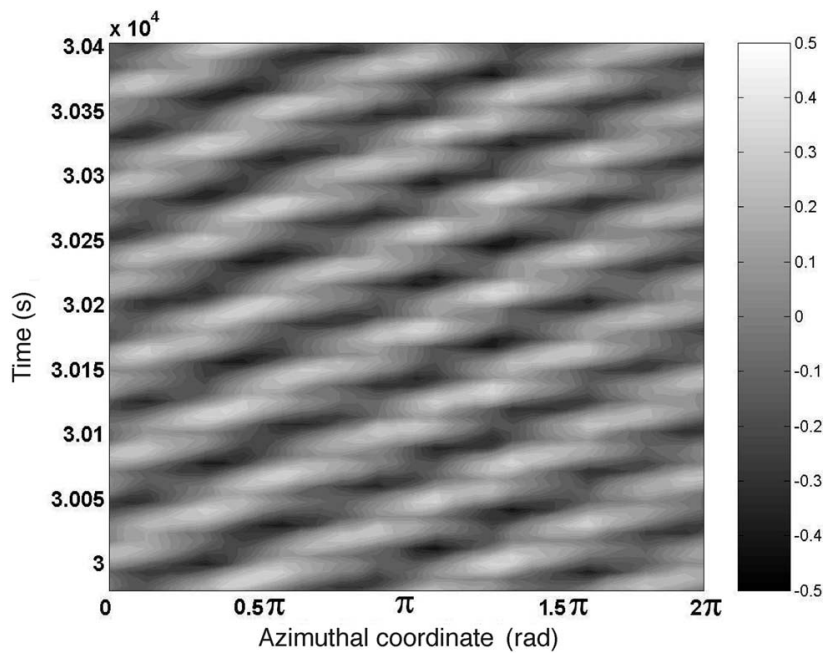

FIG. 8. Azimuth-time temperature contour plot of a traveling wave with $m=3$ at midheight and midradius, showing amplitude vacillation. $\left(\mathrm{Ta}=8.23 \times 10^{5}\right.$ and $\left.\Theta=0.593\right)$ at midheight and midradius. 


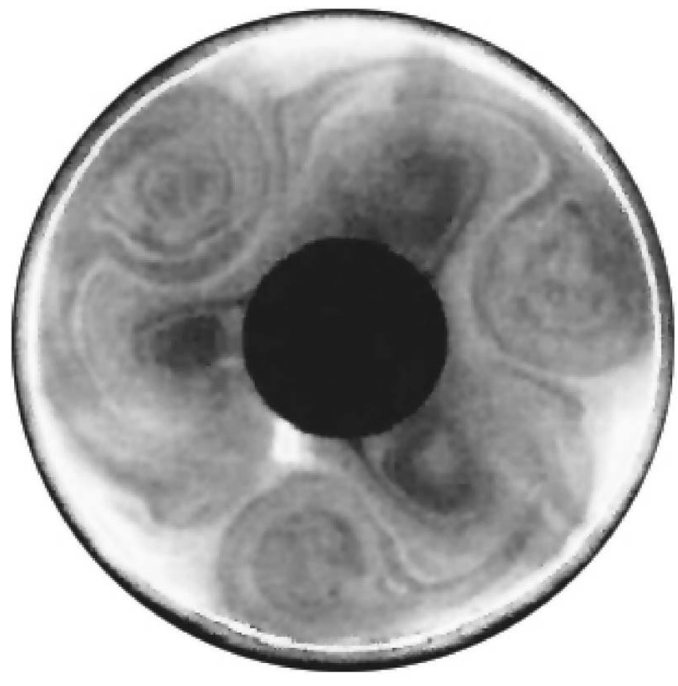

FIG. 9. Visualization of a $3 \mathrm{AV}$ wave $\left(\mathrm{Ta}=8.23 \times 10^{5}\right.$ and $\Theta=0.593)$ at midheight.

Such large temperature amplitudes are unusual in experiments with high Pr liquids, though not unknown. After a detailed study of the visualization results it was concluded the darker zones in the images correspond to regions where the fluid (or the particles) were traveling downward, in association with relatively clear air entrained from the boundary layer adjacent to the inner cylinder.

The drift frequency, $\omega_{d}$, for this example was approximately $0.0043 \mathrm{~Hz}$ for the temperature measurement experiment and approximately $0.0068 \mathrm{~Hz}$ for the visualization experiment (both results corresponding to the same point in the regime diagram). As discussed by Hignett et al. [13] this difference results from a drag effect associated with the thermocouple ring. This may be because mid-depth and midradius correspond to a region of "westerly" flow, so that the thermocouple ring exerts an "easterly" drag on the flow, decreasing the drift frequency.

Another possibility is that the two flows (the one visualized and the one with the thermocouple ring) do not correspond exactly to the same point in their respective parameter spaces, which are shifted relative to each other because of the presence of the thermocouple ring.

\section{B. Amplitude vacillation}

When the rotation rate was gradually increased, starting from a steady wave state (for almost all the experiments), a transition to large amplitude baroclinic waves with a periodic amplitude modulation or vacillation was found. As a general example of the range of different flows found in the experiments, the case of $\Delta T=5{ }^{\circ} \mathrm{C}$ is presented in Table I. In Fig. 8 an experimental azimuth-time temperature contour map of a large amplitude $m=3$ wave with amplitude vacillation is shown. An example of the time variation of an AV wave is shown in Fig. 3(b), showing a periodic modulation of the wave amplitude. It is notable that the typical sequence of transitions between flow states from a steady wave flow as rotation rate increases is first to an AV state then to MAV, subsequently leading to another steady state but with a higher wave number. This is largely consistent with the results obtained in numerical simulations presented by Randriamampianina et al. [10], and is in contrast with the common observation in high $\mathrm{Pr}$ fluids that an amplitude vacillation flow is the precursor of a steady state with a lower wave number, when the rotation rate is decreased.

Figure 9 shows a snapshot obtained from the flow visualization system for the same parameters as in the flow shown in Fig. 8. In this figure the jet streams are clearly visible. Qualitatively it can be said that the vortices were stronger at the midheight regions than close to the upper and lower boundaries. Again, the centers of the vortices (black regions in the snapshot) closer to the inner cylinder indicated regions where the fluid was traveling downwards as relatively cold, clear air entrained from the inner boundary layer.

Although AV flows were clearly apparent in the temperature time series, it is notable that such vacillations were hardly recognizable in the visualization experiment.

The drift frequency, $\omega_{d}$, with $\Delta T=5{ }^{\circ} \mathrm{C}$ was approximately $0.019 \mathrm{~Hz}$ for the temperature measurement experiment and approximately $0.023 \mathrm{~Hz}$ for the visualization experiment, this time showing relatively good agreement. However, as discussed above, effects of the thermocouple ring caused the drift frequency to be smaller than the one obtained using flow visualization.

The magnitude of the vacillation in the AV and MAV flows is usually quantified by a vacillation index [10], defined as

$$
\eta=\left(A_{\max }-A_{\min }\right) /\left(A_{\max }+A_{\min }\right),
$$

where $A_{\max }$ and $A_{\min }$ are the maximum and minimum amplitudes of the temperature oscillation, respectively. This value is very helpful to delineate the dependence of the strength of the vacillation when variations in the external parameters are applied. The values of this index for the case of $\Delta T=5{ }^{\circ} \mathrm{C}$ are presented in Table I. It can be seen that there is a tendency of the vacillation index to decrease when the rotation rate is increased. This behavior was also seen at other temperature contrasts. Also, it can be seen that $\omega_{d}$ decreases its value when the rotation rate is increased in the region of large amplitude waves. However, this drift frequency suddenly increases in the region of the weak waves (going from low values to high values of rotation rate). On the other hand, for $\omega_{v}$ the frequency increases as $\Omega$ is increased. However, the strength of this vacillation, quantified by the vacillation index, $\eta$, actually decreases as the rotation is increased, following the initial onset of the vacillation, so that the relative intensity of the vacillation decreases, leading eventually to a steady state, but of a higher wave number.

\section{Modulated amplitude vacillation regime}

When the rotation was increased, in the case of $\Delta T$ $=5{ }^{\circ} \mathrm{C}$, the dynamics developed a second oscillation on the wave amplitude. In fact this is a secondary modulation of the vacillation itself, whose frequency, $\omega_{m}$, was always smaller than the vacillation frequency $\omega_{v}$. It is important to note that, in all the cases investigated here, $\omega_{m}$ was always incommen- 


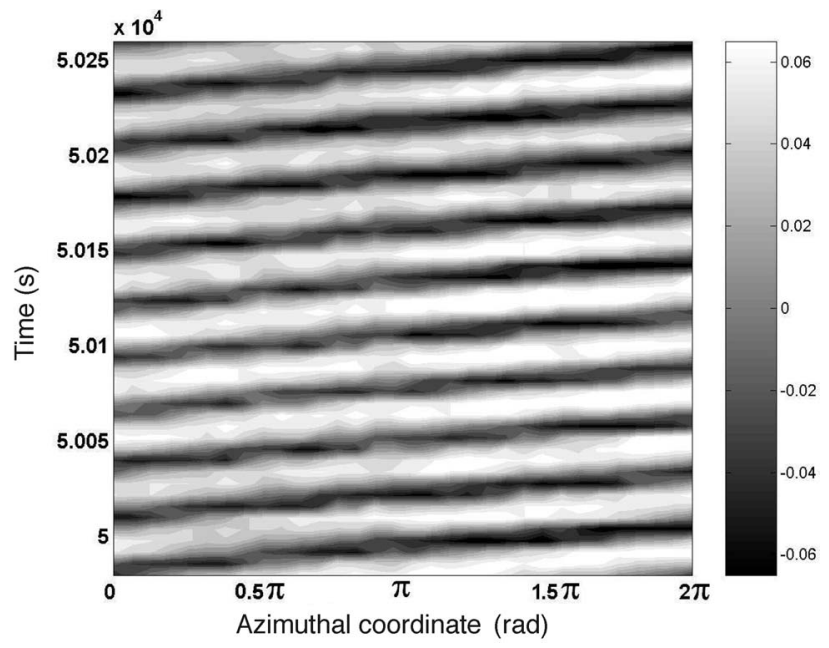

FIG. 10. Azimuth-time contour plot of temperature of a traveling weak wave with $m=1$. Ta $=2.202 \times 10^{5}$ and $\Theta=2.218$ at midheight and midradius.

surate with either $\omega_{v}$ or $\omega_{d}$. Very small variations in the drift velocity as a result of the vacillation and modulation of the wave were also observed, as much as found in vacillations with high Pr fluids. Figure 5(c) shows the typical evolution of the amplitude of a MAV flow. At least two independent frequencies appear to be present in this signal, comprising a long period oscillation modulated by a fast high-frequency oscillation. The wave number spectrum was fairly pure with only one predominant peak at the value of $m=3$. Again, an oscillation on the drift velocity was detected, but it was more complex in this case, because both the AV and MAV frequencies were perturbing the drift frequency of the wave. Unfortunately, principally due to the time limitations on the visualization experiments, but also perhaps because $\eta$ was relatively low $(\leqslant 0.1)$ for all the vacillations encountered in these experiments, MAV oscillations were impossible to detect and measure by visualization.

As shown in Fig. 3(c) and Fig. 5(c), the frequency spectrum of a typical MAV flow consists of three incommensurable frequencies, the drift frequency, $\omega_{d}$, the vacillation frequency, $\omega_{v}$, and the modulated vacillation frequency, $\omega_{m}$. As found by [10] and in [8], MAV flows can be either periodic or chaotic. In this case, with such a pure and well-defined frequency spectrum, the dynamics was concluded to be quasiperiodic. This conclusion was confirmed via a calculation of the maximum Lyapunov exponent (which was $2 \times 10^{-5} \pm 3.1 \times 10^{-5}$ and therefore indistinguishable from zero) [14]. While the existence of stable three-frequency flows is not typical of generic dynamical systems, such a result is possible for systems characterized by certain degrees of symmetry.

The regime diagram presented in Fig. 4 indicates a 3 MAV regime lying between two distinct $3 \mathrm{AV}$ regimes. A careful analysis of the frequency spectra was carried out, in order to find out whether the lowest (in the regime diagram) 3 AV regime could simply be a 3 MAV flow in which one of the frequencies became locked to the drift frequency or with a harmonic of the $3 \mathrm{AV}$ frequency. The results showed, on the contrary, that the lower $3 \mathrm{AV}$ flows actually correspond to

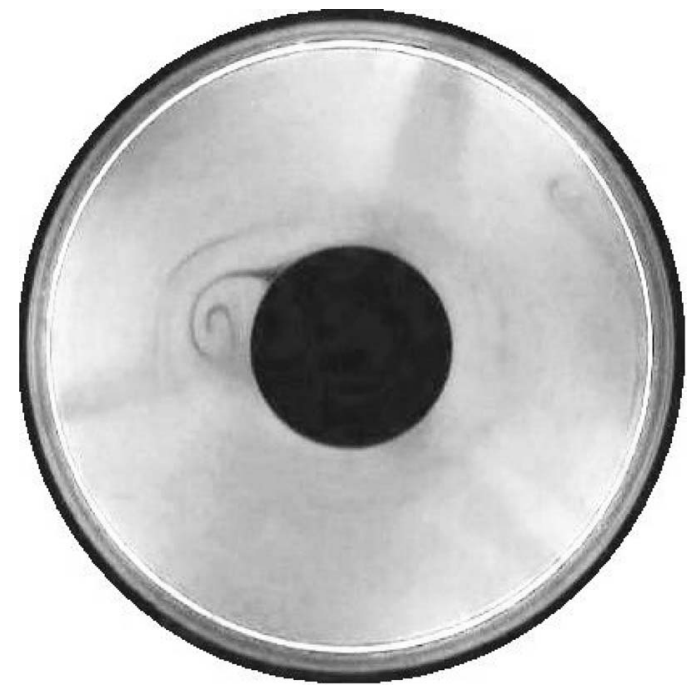

FIG. 11. Visualization snapshot of a weak wave with $m=1$ at $\mathrm{Ta}=2.202 \times 10^{5}$ and $\Theta=2.218$ at midheight.

a distinct flow regime from the upper $3 \mathrm{AV}$ flows (in the regime diagram) and the $3 \mathrm{MAV}$ regime, exhibiting a lower vacillation frequency than the corresponding $\omega_{v}$ of the 3 MAV flow.

\section{Weak waves regime}

As can be seen in the regime diagram in Fig. 4 a substantial part of the parameter space investigated (principally towards the upper left) was found to be occupied by a zone where waves with a very small (but crucially nonzero) amplitude were found. They were typically characterized by having amplitudes less than 5\% of the applied temperature gradient and by having relatively large drift frequencies when compared to the parametrically adjacent, large amplitude baroclinic waves. Most of these cases were found to be waves with $m=1$ located mostly near the upper left side of the regime diagram, although there is a small zone in the upper right side of the regime diagram (Fig. 4) where weak waves dominated by $m=2$ were also found. It is notable that the boundary between the weak and large amplitude wave regimes is clearly delimited, as the shaded contours show.

They are also characterized by having a large drift velocity, in contrast to the nearby large amplitude waves. This drift velocity, however, decreases when the rotation rate is decreased. Nevertheless, as apparent in Table I, the abrupt change in $\omega_{d}$ as the weak-strong wave boundary is crossed is remarkable. For the $\Delta T=5{ }^{\circ} \mathrm{C}$ example, this change is by a factor of more than 10, from 0.0043 to 0.0421 , when the rotation rate is decreased across the transition from $2 S$ to $1 W$.

In Fig. 10 an azimuth-time contour plot of temperature at midheight and midradius shows a propagating weak wave with $m=1$. The temperature difference applied in this case was $\Delta T=5{ }^{\circ} \mathrm{C}$, and the amplitude of the wave is approx $0.045 \pm 0.002{ }^{\circ} \mathrm{C}$. An extract illustrating the temporal evolution of the amplitude of this wave is shown in Fig. 5(d), where it can be seen that its amplitude remains fairly constant in time. The small variations apparent in Fig. 5(d) are 


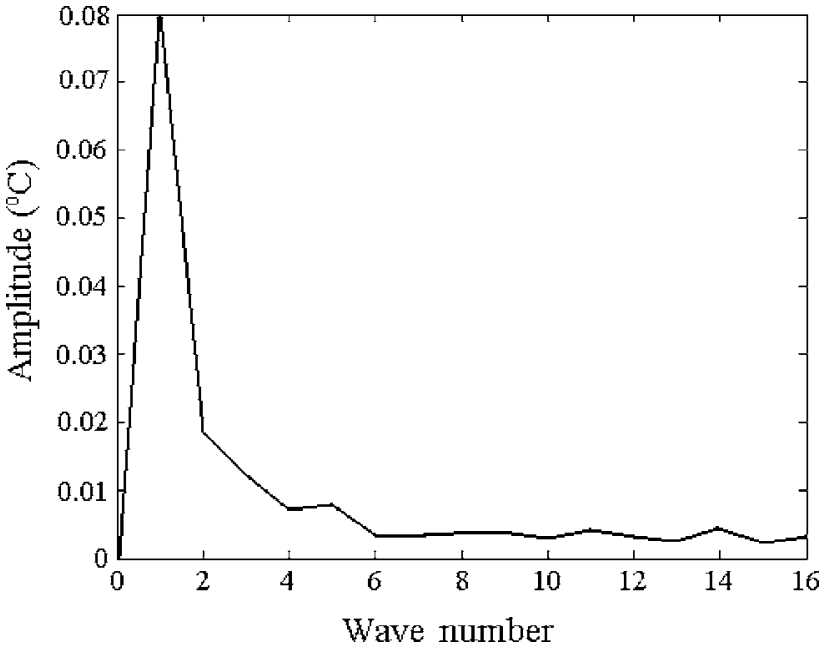

FIG. 12. Time-averaged wave number spectrum of a weak wave with $m=1$ at $\mathrm{Ta}=4.250 \times 10^{5}$ and $\Theta=4.589$.

consistent with the unavoidable level of experimental measurement noise, which is more apparent in this case due to the small temperature fluctuations produced by the weak waves.

One of the most important results of the visualization experiments was the confirmation that the weak waves are a real physical phenomenon, and not an artifact or a misleading interpretation of the results from the temperature measurements. They are clearly visible in the visualization snapshot and movies. It was also concluded that these weak waves are different in character to the ones described in [12]. The latter suggested that it was possible to see weak waves in some experiments (with high Prandtl number) near the boundary in parameter space between axisymmetric and nonaxisymmetric flows, and that they develop with a maximum in amplitude just near the upper or lower horizontal walls of the annulus with no presence in the interior. In this air-filled annulus experiment, however, the weak waves were visible at all heights, and as observed in the visualization results, they have manifestation at all radii. However, they were

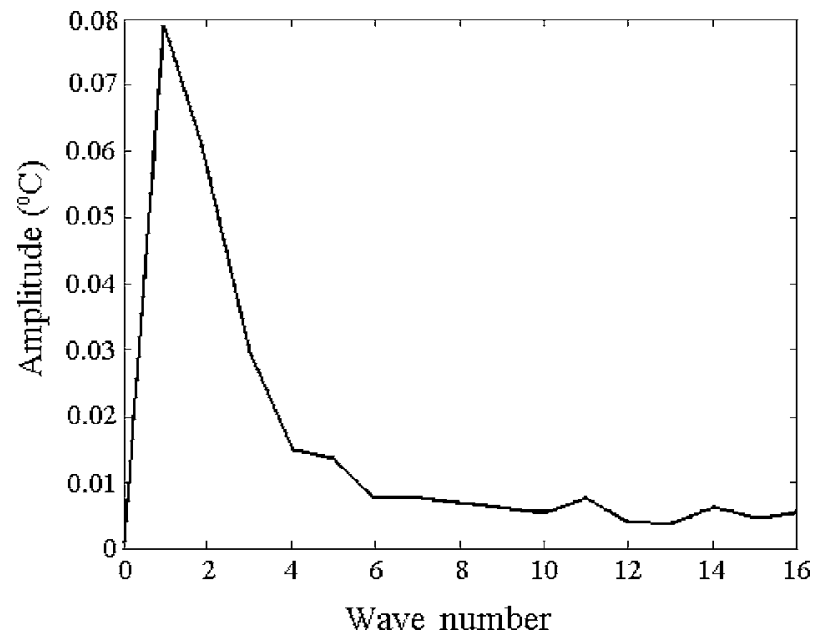

FIG. 13. Time-averaged wave number spectrum of a weak wave with $m=1$ at $\mathrm{Ta}=8.229 \times 10^{5}$ and $\Theta=2.373$.

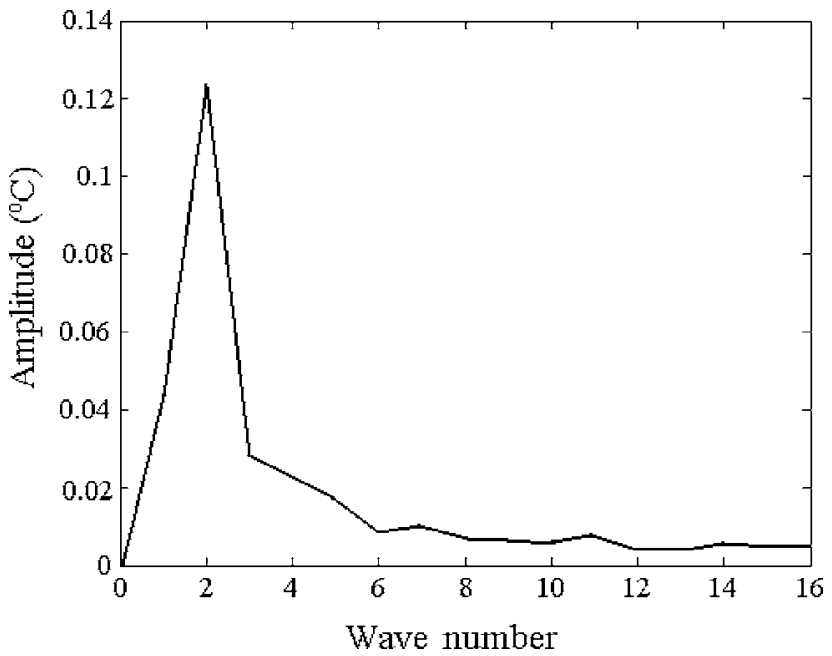

FIG. 14. Time-averaged wave number spectrum of a weak wave with $m=1$ at $\mathrm{Ta}=1.025 \times 10^{6}$ and $\Theta=1.905$.

clearest at mid-depth, and were most prominent close to the inner cylinder. One of the clearest images obtained of this type of wave can be seen in Fig. 11. For this particular example, at $\mathrm{Ta}=2.202 \times 10^{5}$ and $\Theta=2.218$, the main vortex in the wave was traveling clockwise around the inner cylinder while the rest of the fluid was traveling anticlockwise.

As a way of comparison between the transition between large amplitude baroclinic waves and weak waves we will analyze in more detail the experiment with $\Delta T=20^{\circ} \mathrm{C}$. As shown in the regime diagram, Fig. 4, all the flow types found within this condition were of the weak waves type, either with $m=1$ or $m=2$. Also, it can be seen that there is a small region, possibly where the wave number transition occurs, where a mixed $1-2 W S$ was observed. The flow in this region was characterized by having a nonpure wave number spectrum, i.e., the amplitude of $m=2$ was not negligible. This is clearly seen in the wave number spectra for three different cases from this run, as shown in Figs. 12-14. Figure 12 shows the spectrum in the region of $1 W S$, Fig. 13 in the mixed region of $1-2 W S$, and Fig. 14 in the region of $2 S W$

It is important to remark that little or no unsteadiness was observed to within the limitations of the noise level in our experiments (around $\pm 0.0022^{\circ} \mathrm{C}$ ). Even with the mixed wave number states found and presented above, the amplitudes of the individual components were found to be constant to within $\pm 0.0031{ }^{\circ} \mathrm{C}$ in all the cases examined.

\section{DISCUSSION}

In this study we have presented an experimental study of baroclinic waves developed in an air-filled thermally driven rotating annulus flow. The regime diagram covered by the experiments (Fig. 4) shows a great variety of distinct flow regimes, including fully developed steady waves, amplitude vacillations, modulated amplitude vacillation, and so-called "weak waves" (Figs. 10 and 11). Some of the results qualitatively corroborated the numerical simulations described by Randriamampianina et al. [10] regarding the progression of the transitions. This progression flowed from a steady wave 
to an amplitude vacillation regime when the rotation rate (and Ta) was increased, leading eventually to a state with a higher wave number when the rotation rate was further increased. This is in contrast to that found in experiments with liquids of $\operatorname{Pr} \gg 1$. This suggests, as proposed by Randriamampianina et al. [10], that the Prandtl and Taylor numbers play important roles in the nature of transitions or bifurcations to AV and MAV states and an elucidation of the nature of these roles should be an object of further study in theory and models. It is notable that the weak wave behavior has not yet been reproduced by the numerical models described by Randriamampianina et al. [10].

The authors are not aware of a theoretical model that fully describes the effects of the Prandtl number on the dynamics of the flow in the annulus experiment. This aspect clearly deserved more attention in future work. However, with the results of this investigation, it can be stated that the Prandtl number must be playing an important role because it is the only important parameter that was changed in our air-filled annulus experiment.

It is also important to remark that no hysteresis was found in the present experimental results, again in contrast with the results of studies using $\operatorname{Pr} \gg 1$, though in contradiction to the numerical results of [10]. However, this may be in part an artifact of inadequate resolution in parameter space in our current experiments, which should be investigated further in future experimental work.
We also report observations of a traveling weak wave regime, which, as mentioned above, has not yet been reproduced in recent numerical simulations [10] and are very different to the weak waves previously reported by Hide and Mason [12]. The main difference is that, in our case, the weak waves have a detectable amplitude at all heights and over a large region of the parameter space. The weak waves described in [12] were only detectable near one of the horizontal boundaries and were confined to specific regions (in parameter space) close to the transition between axisymmetric flows and nonaxisymmetric flows at relatively high Ta. This difference may point to a different physical origin for the two instabilities, the former (air-filled cavity results) perhaps suggesting a barotropic origin for the weak waves while the latter (in high Pr fluid experiments) arising from fundamentally baroclinic instabilities in association with interior potential vorticity gradients [12], potentially opening a new issue in the study of baroclinic and/or barotropic instability.

\section{ACKNOWLEDGMENTS}

A.A.C.P. acknowledges the support of a Dorothy Hodgkin Scholarship administered via the UK Natural Environment Research Council, and to the SEP. We are grateful to Dr. W.-G. Früh, Dr. A. Randriamampianina, and Dr. P. Maubert for insightful discussions on the interpretation and analysis of our experiments.
[1] D. Andrews, An Introduction to Atmospheric Physics (Cambridge University Press, Cambridge, 2000), pp. 140-153.

[2] M. Collins, S. R. Lewis, P. L. Read, and F. Hourdin, Icarus 120, 344 (1996).

[3] J. R. Holton, Dynamic Meteorology, 4th ed. (Elsevier Academic Press, New York, 2004), Chaps. 5, 8, and 10.

[4] W.-G. Früh and P. L. Read, Philos. Trans. R. Soc. London, Ser. A 355, 101 (1997).

[5] R. Hide and P. J. Mason, Adv. Phys. 24, 47 (1975).

[6] R. R. Pierrehumbert and K. L. Swanson, Annu. Rev. Fluid Mech. 27, 419 (1995).

[7] P. L. Read, M. Collins, W.-G. Früh, S. R. Lewis, and A. F. Lovegrove, Chaos, Solitons Fractals 9, 231 (1998).
[8] P. L. Read, M. J. Bell, D. W. Johnson, and R. M. Small, J. Fluid Mech. 238, 599 (1992).

[9] J. S. Fein and R. L. Pfeffer, J. Fluid Mech. 75, 81 (1976).

[10] A. Randriamampianina, W.-G. Früh, P. L. Read, and P. Maubert, J. Fluid Mech. 561, 359 (2006).

[11] W. W. Fowlis and R. Hide, J. Atmos. Sci. 22, 541 (1965).

[12] R. Hide and P. J. Mason, Geophys. Astrophys. Fluid Dyn. 10, 121 (1978).

[13] P. Hignett, A. A. White, R. D. Carter, W. D. N. Jackson, and R. M. Small, Q. J. R. Meteorol. Soc. 111, 131 (1985).

[14] H. Kantz and T. Schreiber, Nonlinear Time Series Analysis (Cambridge University Press, Cambridge, 2002), Chap. 5. 